\title{
Physiological Noise Suppression for Functional Magnetic Resonance Imaging by Normalized Least Mean Square Adaptive Filtering
}

\author{
Ing-Jye Huang*, Kai-Hsiang Chuang*, Yi-Jui Liu*, Fu-Nien Wang*, \\ Cheng-Yu Chent, Hsiao-Wen Chung*
}

*Dept. of Electrical Engineering, National Taiwan University, Taipei, Taiwan, R.O.C.

†Dept. of Radiology, Tri-Service General Hospital, Taipei, Taiwan, R.O.C.

\section{Introduction}

Functional magnetic resonance imaging (fMRI) has been widely employed as a noninvasive, radiation-free and high spatial-temporal resolution neuroimaging tool. However, imterfercnec could mask out the presence of functional activities owing to inherently low sensitivity of the functional signal. Examples include physiological noise duc to cardiac or respiratory pulsation, which has been shown to cause signal fluctuations in certain brain areas. In this study, we propose the application of a normalized least mean square adaptive filter (NLMSADF) (3) for a suppression of the physiological noise using its learning nature.

Methods and Materials

NLMSADF (Fig.1) has been widely used for its computational simplicity. By minimizing the mean square error, the filter coefficient adaptation is given by:

$$
\begin{aligned}
& W(n+1)=W(n)+\frac{\beta}{|R(n)|^{2}} e(n) r_{1}(n) \\
& e(n)=d(n)-y(n) \\
& y(n)=\mathbf{W}(n) \mathbf{R}_{s}(n) \\
& R,(n)=\left\{r,(n), r_{1}(n-1), \ldots r,(n-N+1)\right\}^{r} \\
& W(n)=\left\{w_{0}(n), w_{1},(n) \ldots, w_{*},(n)\right\}^{\gamma}
\end{aligned}
$$

where

$d(n)$ : input signal, the combination of desired signal $s(n)$ and primary noise $r_{0}(n)$.

$r_{1}(\mathbf{n})$ : reference noise, correlated with $r_{n}(\mathbf{n})$.

W(n): NLMSADF's coefficients.

$e(n)$ : the estimated desired signal $s^{\prime}(\mathbf{n})$ if $s(n)$ is uncorrelated with $r_{1}(\mathbf{n})$

$\beta$ : convergence factor.

The convergence factor $\beta$ was determined empirically to be 0.02 , and order of filter to be 7 in this study.

Simulation

The synthetic signal was constructed as follows:

$$
S(n)=S_{-} \text {act }(n)+\operatorname{Noise}_{1}(n)+\text { Noise }_{2}(n)
$$

where

$$
\text { S_act (n):activationsignal }
$$

Noise $_{1}(\mathbf{n})$ : physiological noise. In this simulation, only respiratory noise was included

Noise 2(n): Gaussian white noise.

The reference noise is simulated to be proportional to the physiological noise. The $\mathrm{SNR}_{1.2}$, defined as the standard deviation of S_act over the standard deviation of Noise 1,2 , ranged from 1 to 4 . For cach SNR, 1000 repetitions werc conducted to obtain the normalized noise power suppression defined by:

$$
\text { NNFS }=\frac{\mid \text { ertimated } \text { Nowse }_{1}-\text { Noise }\left._{1}\right|^{2}}{\mid \text { Notse }\left._{1}\right|^{2}}
$$

MRI Experiment

Data from two healthy young male subjects were included. Single-slice gradient echo EPI was acquired repeatedly $(400$ time frames), with $T R / T E=250 / 35 \mathrm{~ms}$, matrix $\times 64, F O V=22 \mathrm{~cm}$, and slice thickness $=4 \mathrm{~mm}$. All experiments were conducted on

\begin{tabular}{|c|c|c|}
\hline 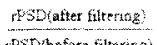 & SulbectI & Suthoect? \\
\hline Carthat & $0.9 \pm 0.11$ & $070 \pm 013$ \\
\hline Respiratory & $0.1 \% \pm 0.13$ & (1) $\alpha(x+1)$ \\
\hline
\end{tabular}
a Bruker MedSpec 3T whole-body system (Ettlingen, Germany). The extraction of reference noise from the acquired images was described in (4). rPSD was defincd as the respiratory or cardiac noise power over that of the entire spectrum obtained from each pixel's time course. Only pixels whose rPSDs exceed 0.08 were used for evaluarion. Results

The performance of NLMS adaplive filter under various levels of SNR is demonstrated in figure 2 and figure 3. IPSD improvements of the two subjects are shown in Table 1.

\section{Discussions}

From the simulation results, we show that NLMS $\triangle \mathrm{DF}$ can reduce nonstationary physiological noise to an acceptable level depending on the noise level. As expected, the proposed algorithm performs better under lower Gaussian white noise. In terms of physiological noise, it is interesting to note that the NLMSADF particularly works well under low SNR conditions. This is because as SNR gets higher, extraction of the reference signal becomes more difficult under nonstationary situations. In fact, it may not be necessary to use an adaptive filter when there exhibits sufficient SNR. We conclude that the efficacy of physiological noise suppression using NLMS adapive filter is comparable with previous study (1) and is particularly suited for low SNR applications.

\section{References}

1. Hu X et al. MRM, 37:877, 1997.

2. Buonocore MH et al. MRM, 38:456, 1997

3. Widrow B et al. Adaptive Signal Processing, Prentice-Hall, Cliffs, 1985.

4. Chuang et al. Proc. ESMRMB, 2000. 